\title{
Hémorragie intra-buccale après avulsions dentaires, point d'appel clinique d'un angiosarcome du sinus maxillaire : à propos d'un cas
}

\author{
Saricassapian B, Lecompte JC, Pommepuy I, Garmi R. \\ (Centre Hospitalier de Guéret, 23000 Guéret)
}

Les sarcomes sont des tumeurs rares, complexes et hétérogènes qui se déclinent en plus de 50 soustypes différents (Classification OMS). Le diagnostic ne peut être établi avec certitude qu'après confrontation multidisciplinaire : clinique, imagerie, anatomopathologie, immunohistochimie. L'angiosarcome, classé dans la catégorie des sarcomes des tissus mous, est une tumeur maligne, agressive et rare (1\% des sarcomes) (Kuffer et al. 2009) (Mücke 2010), d'étiologie inconnue, elle touche principalement la personne âgée. La localisation la plus fréquente de l'angiosarcome est la peau et plus particulièrement le cuir chevelu (Favia 2002). Les localisations au niveau de la sphère ORL ne représenteraient que 4\% (Velegrakis 2000) (Triantafillidou 2002), et entre 1 et 2\% pour la cavité buccale. Des cas de localisation parotidienne ont été décrits (Mullick 1997). L'aspect clinique se présente comme un nodule sous muqueux, très adhérent, érythémateux ou bleuté, saignant spontanément ou au contact. II peut être difficile à distinguer de celui d'autres tumeurs (Kuffer et al. 2009) voire de lésions plus courantes (Mücke 2010). Le diagnostic différentiel histologique pouvant être difficile à établir, l'immunohistochimie est d'un apport indispensable, les cellules tumorales exprimant le CD31, le CD34, la vimentine et le facteur VIII (Yang 2010) (Terada 2011).

Le cas rapporté est celui d'une femme de 75 ans, hospitalisée pour des épisodes itératifs d'hémorragie endobuccale consécutive à des avulsions dentaires réalisées 7 jours auparavant. Suite aux tentatives de contrôle local de l'hémostase, la patiente a été orientée par son médecin vers le service des urgences. Les antécédents médicaux notables sont: une hémoptysie survenue 2 mois auparavant ayant nécessité une hospitalisation, un épisode d'épistaxis 8 jours auparavant et des avulsions dentaires en 2 séances distinctes à 5 jours d'intervalle de 16, 17 puis 44,45,46 et 47 (délabrées et mobiles). Son traitement médical en cours comprend : ésoméprazole $40(0-0-1)$ et méprobamate/acéprométazine (0-0-1). Le jour de l'admission (J), I'hémogramme révèle : un taux d'hémoglobine à $11,9 \mathrm{~g} / \mathrm{dl}, 242000$ plaquettes/mm3, aucun trouble de l'hémostase. A l'examen clinique, il est noté une tuméfaction jugale à droite, un saignement gingival « en nappe » et la présence d'un caillot en regard du site de 47. Les techniques de compression locales permettent de stopper le saignement transitoirement. La patiente est admise dans le service d'hématologie pour surveillance et investigations. $A J+1$, la reprise du saignement sur le site de 16 et 17, nécessite une nouvelle tentative de contrôle de l'hémostase sous anesthésie locale. La biopsie d'un tissu d'aspect bourgeonnant en regard de l'alvéole de 47, est réalisée dans le même temps en vue d'un examen anatomopathologique. Les examens radiologiques standard demandés (panoramique dentaire et incidence de Blondeau), révélant l'existence d'une opacité du sinus maxillaire droit, un examen TDM est réalisé. II met en évidence un comblement total du sinus. Devant la reprise du saignement, la patiente est transférée dans un service spécialisé. Les résultats de l'examen d'anatomopathologie évoquent d'emblée la possibilité d'un angiosarcome, ce qui est confirmé par la relecture des lames et l'immunohistochimie. Devant la persistance de I'hémorragie une hémi-maxillectomie droite a été réalisée à visée carcinologique et hémostatique.

SARICASSAPIAN Bernard sarrybernard@wanadoo.fr

This is an Open Access article distributed under the terms of the Creative Commons Attribution License 2.0, which permits unrestricted use, distribution, and reproduction in any medium, provided the original work is properly cited. 